122

\title{
Grobe Fehler \\ vermeiden und das Minimum erfüllen
}

Verantwortliche in Marketing und Vertrieb verfolgen ein Ziel: Mit ihren Produkten und Dienstleistungen wollen sie die Bedürfnisse der Kunden besser erfüllen als die Mitbewerber. Dasselbe Ziel zu haben bedeutet aber nicht, den gleichen Weg einzuschlagen.

Christian Belz, Michael Reinhold 
Normalerweise befassen sich Manager damit, wie sich Lösungen in Marketing und Vertrieb optimieren lassen. Oft wäre es aber realistisch und weit ökonomischer, einfach größere Fehler zu verneiden oder Beschränkungen zu akzeptieren. Es gilt, verschiedene Blicke auf dieselbe Aufgabe zu werfen.

\section{Falsche und richtige Denkmuster}

Dieser Beitrag zeigt Praxisfalle, Ansatz und Beispiele. Angewendet wird die Sichtweise des Minimalmarketings am internationalen Vertrieb. Anhand von Beispielen ertäutern wir die falschen oder richtigen Denkmuster.

1. Marketing für Suchmaschinen. Google ist die führende Suchmaschine im Internet. Gemäß einer Aussage von Verantwortlichen möchte Google dem Kunden eine Antwort auf seine Frage liefern, noch bevor er diese fertig formuliert hat. Ein immenses Nutzenversprechen. Wie wird dieses eingelöst? Auf die Eingabe des Begriffs ,Marketing“ bekommen wir "ungefähr 230 Millionen Ergebnisse (in 0,32 Sekunden)“. Ist die Anzahl der Ergebnisse fur den Kunden relevant? Nein, solange überhaupt einige Ergebnisse erscheinen. Spielt die Zeit eine Rolle? Nein, solange sie,gefühlt' kurz ist. Sind diese beiden Zahlen überhaupt korrekt? Das weiß nur Google und es spielt überhaupt keine Rolle, weil nicht nachprüfbar. Spielt die Qualität der Ergebnisse eine Rolle? Klar ja, aber nur ganz am Anfang der Liste, weil kaum jemand die restlichen fast 230 Millionen Antworten durchliest. Damit reduziert sich die Aufgabe für den Anbieter auf eine Variable und eine Randbedingung. Die zu optimierende Variable ist "Wenige, aber gute Antworten“ zu Beginn des Suchprozesses. Die „Güte" der Antworten wird von Google darüber bestimmt, ob der Kunde eine Auswahl überhaupt anklickt und wie lange er auf der entsprechenden, landing page verweilt. Die Randbedingung lautet: Die Bezahlinserenten müssen gelistet sein. Es gilt somit, grobe Fehler zu vermeiden. Beispielsweise darf kein Prozessabbruch durch den Kunden erfolgen, weil die Ergebnisse grundfalsch oder unpassend sind. Zudem ist der Kundenerfolg messbar.

2. Beispiel für Recommender Engines - Netflix: Besonders im Internet ist diese Denkweise üblich. Interessant ist das Beispiel der Empfehlungsplattform Netflix für Videos-on-demand. Diese ist in den USA stark verbreitet und startet derzeit mit der Markteinführung in Deutschland und der Schweiz. Netflix verwendet einen kollaborativen Filter-Algorithmus, um dem Kunden basierend auf seinen bisherigen Ratings und den Ratings anderer Nutzer den bestgeeigneten nächsten Film zum Kauf vorzuschlagen. Auch die Kunden von Amazon kennen alle den berühmten Satz: "Customers Who Bought This Item Also Bought". Ziele dieser Händler sind der Neu- und Wiederkauf sowie das Up- und Cross-Selling. Die Transaktion spielt sich im Internet ab und ist daher gut messbar und zu verfolgen. Der Erfolg von Netflix hängt unter anderem auch von der Qualität der Empfehlungen ab.

Auf Grund dieser Oberlegung schrieb Netflix am 1. Oktober 2006 eine Million US-Dollar Preisgeld aus. Es sollte diejenige Gruppe bekommen,

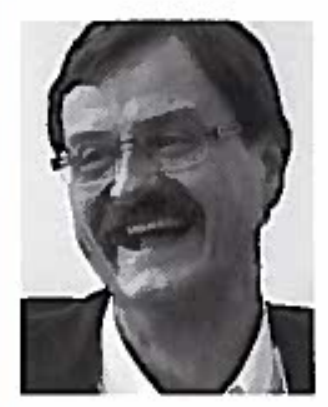

Prof. Dr. Christian Belz ist Ordinarius für Marketing an der Universität St. Gallen tand Direktor am Institut für Marketing

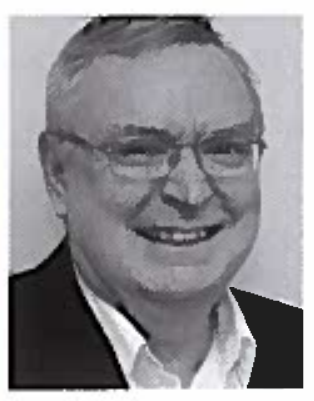

\section{Dr. Michael Reinhold}

ist Lehrbeauftragter der Universität St. Gallen und leitet am Institut für Marketing die Bereiche Hightech Marketing und Live Communication.

Christian Belz

Universität St. Gallen, St. Galien, Schweiz

E-Mail: christian.belzeunisg.ch

Michael Reinhold

Universität St. Gallen, St. Gallen, Schweiz

E-Ma l: michael.reinhold@unsg.ch 


\section{Kerngedanke 1}

\section{Unternehmen wollen ihre} Lösungen im Marketing und Vertrieb meistens optimieren. Häufig würden sie sich dabei erfolgreicher darauf konzentrieren, grobe Fehler zu vermeiden oder Minimalkonzepte zu entwickeln. die zuerst einen un mindesters z.chn Prozent verbesserten Fmpfehlungsalgorithmus im Vergleich zum hauseigenen Algorithmus "Cinematch" prïsentierte. Der Wettbewerb hatte mathematisch sauber uberprübare Konditionen. Der Preis ging am 21. September 2009 an das Team "BellKor's Pragmatic Chaos" bestehend aus Forschern von AT\&'T.

Verwendel Netflix diesen Algorithmus heute? Die Antwort von Netflix ist nein, jedenfalls sicher nicht online und in realtinse. Der Grund dafur liegt darin, dass die positive Verbessertung der Vorschläge fur den Nutzer kaum eine Rolle spjelt. Entscheidend ist es vielmehr, dass die gesamte user experience der Netflix-Mitglieder optimiert wird.

3. Recommender Engine fiir Geschenkartikel - Dayzzi: Typisch ist auch das Beispiel einer Empfehlungsplattform für Geschenke, die zusammen mit Industrie- und Hochschulpartnern am IfM entwickelt wurde. Um diese kimpfehlung zu optimieren, befrigt das System die Nutzer nach bis 31 verschiedenen Merkmalen der Geschenke-Empfanger und der -Situation (vom Alter der zu Beschenkenden bis zum Geschenk für ein Firmenjubiläum). Es gilt daher, aus ca. 540 Millionen möglichen Kampagnen und einem Fundus von mehr als 50.000 Artikeln der beteiligten Firmen einige passende und kreative Vorschläge zu machen. Die Arbeit mit der Plattform zeigte: Diese positive Kingrenzung spielt kaum eine Rolle. Entscheidend ist es jedoch, dass die Nutzer keine offensichtlich falschen Vorschläge erhalten; etwa ein Messerset für den Vielflieger oder für Kinder. Dann brechen sie den Prozess sofort ab. Mehrere erfolgreiche Verkäufer im Werbemittelmarkt haben darauf hingewiesen, dass der Internet-Recommender eigentlich nichts anderes macht, als ihr Verkaufsgespräch mit dem Kunden 1:1 abzubilden.

4. Mikroanalysen der Verkaufsprozesse beim Kunden: Ähnlich gehen wir bei gründlichen Analysen der Kaufprozesse von Kunden vor. Damit wird erhoben, an welchen Stellen in detailliert erfassten Kaufprozessen der Kunde häufig abbricht. Hier wird dann mit Maßnahmen angesetzt. Ziel ist zwar immer noch der Kaufabschluss, jedoch als logische Folge richtig angesetzter Maßnahmen auf dem Weg dorthin.

\section{Fehler vermeiden und Minimalziele erfüllen}

Grobe Fehler zu vermeiden, um den Kundenprozess in Gang zu halten, scheint in der On- und Offline-Welt erfolgsversprechend, wenn auch im Bereich des Internets selbstverständlicher angewendet. Bezogen auf die Marktbearbeitung und besonders Verkaufsgespräche befassen wir uns dann damit, was Kunden dazu führt, ihre Beziehung mit dem Anbieter abzubrechen. Das entspricht auch vielen persönlichen Erfahrungen. Der Kunde stellt keine sophistizierten Ansprïche an eloquente Verkaufsgespräche und Unterstützung. Er akzeptiert kleinere Probleme, schon weil er den Aufwand für die weitere Suche und zusätzliche Gespräche scheut. Es gibt aber einige grobe Verstöße für ihn. Er empfindet sie als Zumutung und steigt aus. Beispielsweise wentı er dem Verkäufer seine Situation schildert und den Bedarf um- 
reisst, der Verkäufer aber auf ganz anderes eingeht. Auch können mangelhafter Anstand und Druck Richtung Abschluss rasch zum Abbruch führen.

Ein anderer Fall ist gegeben, wenn Optimierungen chronisch an ihre Grenzen stoßen und illusionär werden. Beispielsweise lässt sich beliebig viel von Personen mit Kundenkontakt fordern, nur lassen sich einige Ansprüche mit den verschiedenen, bestehenden Mitarbeitern nicht einlösen. Hier mit Minimal-Ansätzen zu arbeiten, ist ein guter Weg.

Die Bet rachtung umzukehren, kann in manchen Fällen ergiebig sein. Die Tabellen 1 und 2 zeigen dazu mehrere Beispiele aus der realen und der virtuellen Welt.

Trotz der Unterscheidung: Die Trennlinie zwischen Fehlervermeidung und Minimalzielen lässt sich teilweise nicht klar ziehen. Das Erkennen und Setzen von Minimalzielen ist immer dann von Bedeutung, wenn Ressourceneinschränkungen vorliegen, sei es im Geld, in der Zeit oder im geschulten Personal. Als Beispiel vertiefen wir den Ansatz zur letzten Frage in Tabelle 2.

\section{Minimal- und Standby-Marketing im Vertrieb}

Typisch zeigt sich die Problematik im internationalen Vertrieb. Selbst für Konzerne ist es risikoreich, neue Länder zu erschließen. Häufig werden die Businessplane deshalb angepasst und die Zeit zum Break Even verdoppelt oder verdreifacht. Potenziert wird diese Herausforderung für Nebenmärkte oder kleinere Anbieter.

Umso mehr gilt es, zwischen aufwendiger Optimalvariante und Minimalvariante zu unterscheiden.

Mit Minimalmarketing bemühen sich Unternehmen in spezifischen Ländern einzusteigen oder selektiv attraktive Aufträge zu erreichen, ohne aufwendige Infrastrukturen vor Ort bereitzustellen. Oft wird damit ein relativ

\section{Zusammenfassung}

In Marketing und Vertrieb suchen Anbieter in vielen Bereichen nach optimalen Lösungen. Oft bleiben definierte Konzepte jedoch illusionär und lassen sich kaum durchsetzen. Die Autoren argumentieren, dass demgegenüber Minimallösungen bereits viel bewirken können.

\section{Tab. 1 Abbruch vermeiden Optimierung}

Super-Verkaufsgespräche: Wie führen unsere Verkäufer ein kompetentes und sympathisches Verkaufsgespräch mit Kunden?

Attraktion: Wie wird unsere Website noch attraktiver gestaltet? Beisplelsweise durch Angebote und Ablenkungen branchenfremder Anbieter und sozialer Medien sowie die Integration der Breaking News und des lokalen Wetterberichts? Womit bieten wir noch mehr Unterhaltung, vom YouTube-Filmchen hin bis zum eigens komponlerten Song am letzten Firmenanlass? Haben wir ihn bei der Anmeldung auch nach seinen sonstigen Vorlleben befragt?

Unterhaltung: Wie gelingt es uns, dem Kunden den attraktivsten und unterhaltsamsten Aufenthalt auf unserer Website zu vermitteln und möglichst viele "likes" einzusammeln?
Abbruch vermeiden

Verkaufsgespräche ohne grobe Fehler: Wann brechen unsere Kunden den Kontakt im Verkaufsgespräch ab und wie vermejden wir diese Fehler?

Nützlichkeit und Zuverlässigkeit: Werden die Kunden auf unserer Website rasch und loglsch durch ihre Ansprüche und Wünsche geführt? Berücksichtigen wir den Großteil aller Zielgruppen? Durch welche Maßnahmen können wir sicherstellen, dass das Anliegen des Kunden - die Customer Journey - rasch und zufriedenstellend abgearbeitet wird? Wie räumen wir die Hürden im Prozess weg. Wo kann der Kunde Hilfe bekommen, wenn er sich auf unserer Homepage nicht mehr zurecht findet?

Effizienz: Wie messen wir die Verweildauer der Kunden auf den sachlichen Inhalten unserer Website? Wie verläuft der Suchprozess des Kunden und wohin wandert er schließlich ab? Wie sind die Konversionsraten im Prozess des Kunden?

Quelle: eigene Darstellung 


\section{Kerngedanke 2}

\section{Anwendungsfelder für Fehler- vermeidung und Minimalkon- zepte sind in Marketing und Vertrieb mannigfaltig.}

passiver Export aus dem Heimland fortgesetzt. Manchmal folgen die Anbieter auch neuen Standorten ihrer Kunden und etablieren dafur eine minimale Infrastruktur vor Ort.

Mit optimierten Vertriebsengagements wollen oft Marktführer und gro Be Unternehmen eine professionelle Infrastruktur und Ressourcen einset zen, um Potenziale in den Märkten zukünftig zu nutzen.

Wahrend sich optimale Vorgehensweisen leicht vorstellen lassen, beschränken wir uns auf das Minimal- und Standby-Markeling, welches at traktive Gelegenheiten im Markt mit minimalem Aufwand nutzen kann.

Aus 473 Aussagen von 204 Führungskräften, zur Frage nach dem Minimalmarketing, ergab sich die Gewichtung in Abbildung 1.

Drei Ansätze erwiesen sich dabei für Minimalmarketing in einem Land als besonders wichtig:

1. Informationsbasis: Voraussetzung ist eine realistische lnformationsbasis, um die Absatzchancen für die Leistungen des Unternehmens fundiert abschätzen zu können. Für besondere Leistungen und Preise im neuen Markt muss der Anbieter vorbereitet sein.

2. Engagierte Kämpfer: Entscheidend ist die Person des Leiters der Niederlassung. Es handelt sich dabei meist um Manager mit starkem Beziehungsgeflecht, breiten Kompetenzen und einer unternehmerischen Kämpfernatur für den Aufbau. Solche Kämpfer rekrutieren auch ein entsprechendes Team. Dabei setzen Unternehmen meist eher Landsleute als Expatriates ein, um die Kosten zu beschränken und rasch den Anschluss an die Landeskultur zu gewinnen.

3. Infrastruktur: Je nach Geschäft braucht es eine grundlegende Infrastruktur, beispielsweise die Logistik, den Service vor Ort oder die Vertretun-

\section{Tab. 2 Minimalziele}

\section{Optimierung}

Know-how-Erweiterung: Wie erweitern wir dle Fähigkeiten unserer Key Account Manager, damit sie die multiplen Kundensituationen erfassen, unser gesamtes Angebotsportfollo einbringen könnęn und komplexe Abläufe bis zu anspruchsvollen Vertragswerken beherrschen?

Formalislertung und Organisation: Wie gelingt es uns, für Key Account Management die komplexe interne Zusammenarbeit mit verschiedenen Stellen der Technik, des Controlling,s des Managements usw. zu kJären? Wie gellngt es, die Rollen und Aufgaben zu definleren?

Ködern der Kunden Im Netz: Was könnten attraktive Preise für unsere Zielkunden in einem Wettbewerb sein? Welche Downloads können wir frei nach der Registrierung anbieten? Wie stellen wir den Link zwischen Kunde und Social Media sicher, um noch mehr über ihn in Erfahrung zu bringen?

Wunschprogramm: Wie gelingt es, neue Märkte optimal zu erschließen?

\section{Minimalziele sichern}

Know-how-Ergïnzung: Wie flankleren wir unsere Key Account Manager, um ihre Lücken im Know-how und in der Kompetenz je nach Situation zu schließen?

Beweglichkelt: Wie gehen wir beweglich mit den spezifischen Großprojekten für Großkunden um, weil sich jedes Projektteam, jedes Kundenprojekt, jede Aufgabe unterscheidet? Wie gelingt es, die Fähigkeiten der aktuell Beteiligten aktiv zu nutzen?

Notwendiges Minimalprogramm: Sind in unserem Web-Auftritt alle Kanäle gelistet, auf denen uns der Kunde wiederfinden kann? Kann er uns über ein Formular direkt anschreiben oder kann er uns anrufen?

Notwendiges Programm: Welche minimalen Voraussetzungen sind für das Unternehmen nötig, um in einem neuen Markt präsent zu sein? 
gen fur Regionen des Landes. Manchmal sind Kooperationen mit ergänzenden Anbietern ergiebig.

Minimalmarketing kann auch er forderlich sein, wenn vorhandene Märkte einbrechen und die Unternehmen trotzdem durchhalten wollen, um für einen neuen Aufschwung gewappnet zu sein.

Kurz: Mit einem minimalen Engagement senken Anbieter ihre Risiken. Allerdings braucht es dazu doch das Niveau, um nicht unter der Wirkungsscliwelle vorzugehen.

\section{Folgerung}

Bei Optimierungen neigen die Verantwortlichen dazu, das Unmögliche zu versuchen. Oft ist die Schadensbegrenzung der realistische und wirksame Ansatz, obschon eher ungewohnt. Für den Kunden müssen unsere Anstrengungen im Vergleich zu Wettbewerbern zumutbar bleiben, das genügt und ist besonders wirtschaftlich. Die Betrachtung ist nicht neu. Schließlich lassen sich zu Chancen immer auch die Risiken eines Unternehmens betrachten. Minimallösungen sind jedoch nicht einfach ein ängstlicher Zugang zu Marketing und Vertrieb. Sie beruhen auf professionellen Prioritäten.

Folgerungen ergeben sich für die Analysen und Lösungen. Zuerst gilt es, die Fehler (etwa in Kundenprozessen) zu erfassen oder Abbruchraten im

\section{Handlungsempfehlungen}

- Im Internet-Marketing ist der Ansatz stärker verbreitet, weil sich Abbrüche gut verfolgen lassen. Lernen Sie im Offline-Vertrieb vom Online-Vertrieb.

- Bestimmen Sie die Felder in Marketing und Vertrieb, bei welchen Optimierungsansätzen Sie bisher scheiterten und Sie sich mit 40-Prozent-Lösungen begnügen mussten.

- Bearbeiten Sie die wichtigen Felder unter dem Blickwinkel von Fehlervermeidung und Minimalkonzepten.

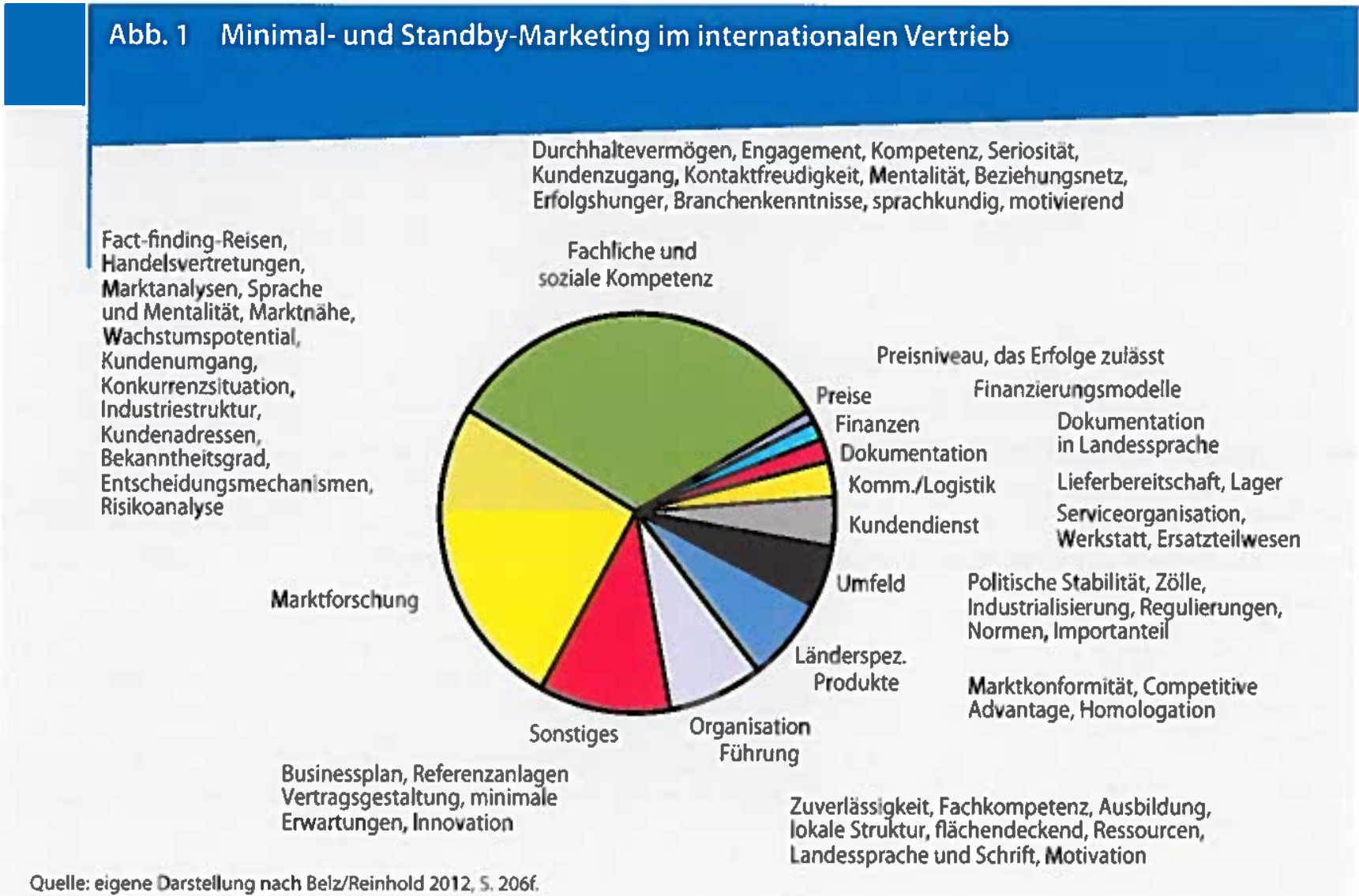




\section{Kerngedanke 3}

\section{Der Wunsch nach Optimierung offenbart meist einen Mangel an Fantasie.}

Prozess zu messen. Für Konzepte ist zu analysieren, was minimale Voraussetzungen des Unternehmens fur den Erfolg sind. Das Niveau zu bestimmen ist anspruchsvoll, denn leicht kann ein minimales Engagement gar keine Wirkung mehr erzielen. Die Lösungen konzentrieren sich darauf, den möglichen Scluaden zu begrenzen. Sie lassen sich weit besser konzentrieren als Optimierungsansätze und vor allem bleiben die Ansätze nicht illusionăr. Schulungen für den persönlichen Verkauf würden beispielsweise durch den "Fehler-Ansatz' umgekrempelt.

Die Anwendungen für den Blick auf Feh]er-Vermeidung oder MinimalLosungen sind im Marketing und Vertrieb unbegrenzt. Nur ist es definitiv schôner über optimale Lösungen zu diskutieren, als lediglich den Schaden zu begrenzen.

\section{Literatur}

[5: ${ }^{*}$ Bel\%, C. (2014): Verbreiterung der Aufgabe des Verkaufs verunsichert die Kunden, in: Sales Management Review Nr. 1, S. 18 -24 (ID: 4999498)

* Belz, Ch. / Reinhold, M.(2012): Internationaler Industrievertrieb, jn: Binckebanck, L.. / Belz, Ch. (Hrsg., 2012): Internationaler Vertrieb, Wiesbaden, S. 6-218 (ID: 3492772)

Rutschmann, M./Helz, Ch. (2014): Reales Marketing, Stuttgart

Abomnenten des Portals Springer fur Professionals erhalten diesen Beitrag in Volltext unter www springerprofessional.de/ID

Zusätzlicher Verlagsservice für Abonnenten von "Springer für Professionals |Vertrieb"

\section{Medium}

$\square$ Online-Artike! (11)

$\square$ Kompakt-Dossier (1)

$\square$ Zeitschriftenartikel (95)

$\square$ Buchkapitel (459)

$\square$ Nachrichten (2)

\section{Sprache}

$\square$ Deutsch (567)

$\square$ Englsch (1)
Von der Verlagsredaktion empfohlen

Rutschmann. M./Belz, Ch.: Titel Beitrag, in: Belz, Ch.: Innovationen im Kundendialog. Wiesbaden 2011, 5.197-222, www.springerprofessional.de/1816256

Biesel, H. H.: Veränderungsprozesse gestalten und umsetzen, in: Bieseli, H. H. Vertriebspower in turbulenten Zeiten, Wiesbaden 2014, S. 77-131, www.springerprofessional. de/5000514 